\title{
SUBSTANTIATING THE DEVELOPMENT STRATEGIES FOR TOURISM IN PROTECTED AREAS
}

\author{
Mirela Costencu \\ "Eftimie Murgu" University of Resita \\ costencumirela@yahoo.com \\ Claudia-Nicoleta Dobrescu \\ "Eftimie Murgu" University of Resita \\ dobrescu_cld@yahoo.com
}

\begin{abstract}
The protected natural areas, irrespective of their classification modality or the motivations they propose, have become more and more attractive for tourists. However, in time, the increasing touristic flows they attract, often developed in an uncontrolled manner, lead to the erosion of the space and the degradation of ecosystems. Developing tourism on sustainable principles, with the appropriate balance of the two functions of a protected area - the scientific and the touristic function - should start from the analysis of this form of tourism and of the development opportunities and limits regarded from the perspective of the external environment, and from the particularisation of the concept "site's tourist reception capacity". Destination areas should meet the economic, social and ecological requirements in competition, so that they observe the integrity of natural resources and of local communities. The upper limit of the number of visits allowed in a protected area is purposefully set at a level below the identified level of accepted use, so that, in the long run, the environment could be able to cover the possible increases occurred in the number of tourists, without subjecting the environment to further harm.
\end{abstract}

\section{Keywords}

protected natural area; touristic flows; SWOT analysis; site's tourist reception capacity; development strategies

\section{JEL classification:}

Q26; M31

\section{Introduction}

Protected natural areas have become important touristic destinations lately, attracting more and more tourists every year. Although the care for nature protection and the declaration of protected areas is centuries old, the scientific grounding of natural areas' role and the awareness of the need to preserve ecosystems are of a more recent date. The first step in this respect was taken by the founding, more than a century ago, of the Yellowstone National Park. The next important step was the creation, in 1948, of the International Union for Conservation of Nature (IUCN), nongovernmental organism under the UNESCO aegis, which gave a remarkable impulse to international solidarity in the environment protection. Gradually, we have witnessed a worldwide generalisation of the states' preoccupation to create protected areas, in view of preserving ecosystems and enabling present and future generations to benefit from their advantages. Thus, as a result of the remarkable growth of protected areas and of the number of national parks and reservations, there are currently 2978 protected areas on the United Nations list, declared within International Conventions. (Smaranda, 2008) 
In the birth act of the first national park- Yellowstone - March $1^{\text {st }} 1872$ - we read the purpose of creating it: "For the benefit and enjoyment of the people". This sentence concentrates the entire policy of nature protection: it is not done to save a species of plants or animals from extinction, nor for the study of the relations existing in nature and isolated in a strictly controlled area from the access viewpoint, but the protection is made for the population. At the beginning of the $20^{\text {th }}$ century, protected areas started to attract visitors' attention, rapidly becoming areas of high touristic affluence and at the same time a true source of profitability. However, the high number of visitors and the implications of this phenomenon trigger a serious issue: ensuring the balance between nature's preservation and deployment of human activities, problem which determines a series of adequate measures in the administration, management and arrangement of national parks, especially the limitation of the number of tourists and the severe directing of touristic circulation.

At the beginning of the $20^{\text {th }}$ century another conception, however, started to gain terrain in relation with the purpose of national parks: the necessity to preserve natural groups, "natural sanctuaries", defined as areas of spontaneous ecological balance, for biologic researches. The notion of "scientific reservation" consequently occurred. This conception lies at the basis of creating the Engadine national park (1914). (Oarcea, 1990). Added to this serious problem represented by the pressure exercised by tourism on national parks, which tends to affect more and more states, a second problem occurs in relation with the purpose of national parks, which can be synthesised as follows: to preserve for the benefit of tourists or to preserve for the benefit of ecologists?

\section{Protected areas regarded as touristic products}

Protected natural areas, grace to their aesthetic, recreational, educational, scientific valences, constitute remarkably attractive touristic destinations, some of them unique in the world. (Tुigu, 2005) Of all categories of protected areas, those that allow their integration into the touristic circuit are national parks. This is due to the complex role played by national parks, role supported by the two functions fulfilled by national parks:

- A scientific function, of conservation of natural resources;

- A touristic function, of satisfying people's cultural, recreational and resting needs

The national park, included into the touristic circuit, can be, at the same time, a component of several types of touristic products: destination of vacations in nature; host of a seminar on ecological themes / of protection of endangered species; onenight stay for a circuit, scene of initiation nature's secretes. Consequently, the national park possesses and may offer diverse touristic products that use simultaneously or alternatively the same technical and material endowment and the same touristic sights. The components of a touristic product are classified into basic elements (accommodation, food supply, transport) and auxiliary elements (balneo-therapy, leisure, sports, educational activities or adventure).

As regards the basic elements, with slight exceptions (the regional, national, traditional specificity) they do not entirely justify the tourists' need for travelling or leaving the residence.

The choice of a touristic destination is determined by:

- Natural factors: geographic location, relief, landscapes, vegetation, fauna, climate

- General factors of human existence and activity, past and present: language, mentality, hospitality, traditions, folklore, culture (religion, art, science), politics, economy. 
- The human element: the local population's attitude towards tourists, providers, administration and representatives of guarding services and public order

- General infrastructure: transports and communications structure and image of settlements, water and energy supply, sewage, telecommunications.

- Touristic equipment: transport, accommodation, food supply, sports activity, entertainment, information.

The motivation underlying the selection of a touristic product is mainly subjective - it depends on the image consumers have created about the product by: publicity, previous uses or previous descriptions of other uses.

If we regard tourism in national parks as a particular form of ecotourism, we distinguish the following possible motivations for choosing this touristic product:

- $\quad$ return to nature - motivation true for all categories (age, gender, profession, social status). It is the result of the need for relaxation, health, physical and spiritual comfort and proves that modern man cannot disconnect himself from the natural life style, and his contact with the natural environment has positive effects on keeping his equilibrium

- knowledge, comprehension and creativity - these are motivations that can be successfully fulfilled in the ambience of national parks, turning vacations into a true process of assimilating new and numerous knowledge and of acquiring skills able to offer tourists personal satisfactions and a sense of utility.

- $\quad$ aesthetic motivations - they result from the need for beauty, order, purity, harmony, naturalness, and they urge most tourists to consider themselves lucky for the possibility to visit attractive spots grace to their picturesque aspect and charm.

- curiosity - it determines large categories of tourists to get in touch, in situ, with all the specific, sometimes unique qualities of an area and to keep sustainable memories from the novel vacations spend in the natural environment.

The "national park" touristic product is not a large-scale touristic product, but bears the mark of originality, novelty, surprise, and constitutes a rich source of satisfaction of needs and thus of motivation. Basic services - components of the touristic product - are necessary, but not determining in choosing the touristic destination. Consequently, we must take into account the other needs of human beings. Thus, the literature considers that we must focus on immaterial qualities (the need for knowledge, initiative, aesthetic needs, creative needs) meant to satisfy secondary needs, much more personalised, of target customers.

From this perspective, the "natural park" touristic product can be considered:

- A true anti-stress therapy

- A place of encounter with wild nature

- Return to origin $\rightarrow$ for the segments of tourists from great urban agglomerations in the strongly industrialised countries, who, because of the multitude and density of their preoccupations, live a stressful life and more and more deprived of reverie elements or possibility to travel into nature

- A real "laboratory" where we can see life and its action.

The "national park” touristic product can satisfy tourists' needs in two ways: $\Rightarrow$ it contains solutions to diverse problems of consumers (the need for relaxation, isolation from noisy life, documentation, satisfaction of hobbies) $\Rightarrow$ it solves certain states of cognitive dissonance, completing the information (from the press, TV, radio, school) and eliminating the tensions between previous pieces of knowledge , apparently contradictory. 
As touristic product " the national park » has turned into a new product, and we may say that at present, by analysing its commercial life cycle, it is in the growing phase. It however determines in time a growth of touristic flows towards the parks. The increasing dimension of touristic circulation leads to the erosion of space, to the degradation of ecosystems. Thus, the dispute occurs between ecologists and tourism organisers that may be synthesised as follows: «To protect for tourists or to protect against tourists ? ». Obviously, it would be ideal to implement a tourism development in protected areas able to balance the two basic functions of national parks: the scientific function and the touristic function. (Costencu, 2007)

\section{Aspects related to the sustainable development of tourism in national parks}

Policy of tourism's sustainable development in national parks is focused first of all on the set-up of these areas, in zones with different protection degrees, with different degrees of accessibility certain areas being delimited as scientific reservations and being included into touristic circuits. Moreover, one of the most radical and efficient measures was to forbid practices considered "noxious". The list of these types of practice differs however from one country to another.

Although we may refer to almost 150 years of experience in the arrangement and development of national parks, there are still some problems, which, for different reasons, have not found their solution yet. First, neither theoretically nor practically, no universal consensus has been reached in defining the concept of "site's tourist reception capacity"; this capacity should be customised in each particular case, and the appreciation of the "optimum" touristic capacity remains extremely subjective. It was found that there may exist negative ecological effects even in the case of a reduced tourists' circulation, and they are more serious by the very fact they do not take a spectacular turn. (Wearing, Neil, 2000) Second, touristic development policies have sometimes taken precedence before the ecological aspect, and numerous compromises have been reached, which, fortunately, have triggered effects only in the peripheral areas.

The concept of "reception capacity", or bearable capacity of a protected area, has at its core the fact that the environment factors set the limits of the population a zone can bear. When these limits are exceeded, the quality of the environment is harmed and consequently its capacity to bear / support a certain population or activity. This goal is reachable, as the biological studies can determine the level of natural resources use in a certain area, setting thus a regularisation of the access to resources. This scientific grounding explains the wide use of this notion as a concept of management in tourism. In this context, the notion contains 3 main elements:

- The biophysic or ecological element - referring to the natural environment;

- The socio-cultural element - related first of all to the host population and their culture;

- $\quad$ The facility element - referring to the visitor's experience;

The reception capacity varies in time and depending on season, and also on a series of factors such as: tourists' behaviour models; facilities' management and design; dynamic character of the environment, change (in time) of the attitude of the host community, etc.

The international practice shows that the "reception capacity" proved less useful than expected. Those involved in the field expected it to reveal exactly "how many are too many?". There are series of different values and "conceptions" about what defines and involves an "unacceptable impact". There are no methods of absolute measurement that may define notions such as "agglomeration" or losses of 
resources”. It is not possible to determine a certain number beyond which unacceptable events (repercussions / consequences) may occur: "in order to prevent the limitation of consumption on very low levels”. People continue to use a certain area for recreational activities, even when they have obvious negative consequences on resources. This is due to the absence of an adequate frame breaking the relation between the wishes and usage / action of the visitor and the management consequences and decisions. (Wearing, 2000) Consequently, the analysis of the occupation capacity was really ignored due to the complexity of its parameters and, although tourism operators are aware of the fact that too many visitors will degrade the environment and will reduce the quality of customers' experience, both from the perspective of entertainment (recreation) and of tourism. There are very few examples where this analysis was used by tourism agents in order to efficiently limit tourism. The solutions to the problem of over-straining the environment and area overagglomeration differ according to the management policy of tourism operators, most being preoccupied by the excessive use of parks and profitableness indicators. One of the most notable uses of the notions of "reception capacity" was introduced to the literature by Butler (1980) who changed the concept of "product lifecycle", applying it to the destination life cycle.

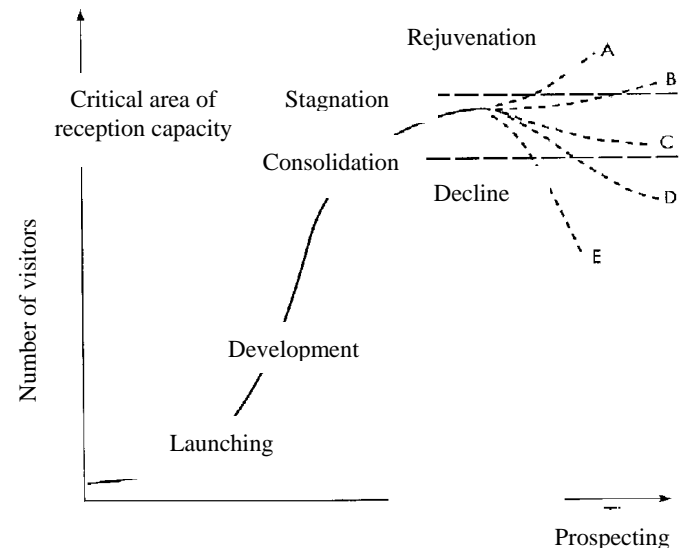

Figure 1 Life cycle of the touristic destination

Source: Butler, RW (1990), The concept of tourist area cycle of evolution: implications for management of resources, The Canadian Geographer, 24

The premise at the basis of Butler's theory consists in the fact that the increase of the number of visitors in an area can be followed by a drop of visitations, as the reception capacity of the respective area is exceeded. It is said that the destination areas go through a relatively uniform transformation in time, from exploitation and launching to consolidation and stagnation, as the material basis of touristic industry changes, in order to receive more tourists and become more competitive. The implications of this theory however makes the managers of destinations to be very careful to any decrease recorded in the ecological destination, and this turns it in the end into the cease of development, as a result of the disappearance of touristic attractions. This is a good example of conceptualisation resorting to the social, ecological and economic implications of tourism in a creating destination frame.

Recently, researchers focused on the derivation of empirical measurements of this evolution of a destination, especially on the level of the natural environment of an island. The usefulness of the concept of "life cycle" has implications in the setting of the limits of reception capacity and social and environment complication of the 
overuse of a touristic destination. Thus, the clear definition of nature and characteristics of a destination's use become a priority.

The general character of the Butler model ensures its applicability in almost any situation in tourism. Reality, as regards this conceptualisation, consists in the fact that, from many perspectives, the concept of life cycle is regarded today in the same manner as it was considered in 1980, when it was conceived, i.e. the reception capacities are still exceeded, immense hotels still exploit to the maximum the resources of the destination. As regards sustainable tourism, it is worth attempting the re-conceptualisation of the Butler model, taking into account the way this cycle would evolve or should evolve, in the ideal hypothetical conditions of sustainable tourism. The literature attempts it, underlining the comparative importance of economic, social and ecological variables in setting a reasonable and long-term level of the reception capacity of an eco-touristic destination. (Fennel, 1999)

If we analyse Romania's situation, we should point out - first of all - that the state's initiatives in this field are not absent. For instance, the law of environment protection defines the general frame of nature preservation based on the strategic principles and elements with the purpose of ensuring a sustainable development. These principles can be synthesised as follows:

- The principle of caution in decision making;

- The principle of preventing ecological risks and damage inflicting;

- The principle of conserving biodiversity and ecosystem specific to the natural environment;

- The "polluter-pays" principle

- Priority removal of pollutants directly and seriously endangering people's health;

- Creation of the national system of integrated environment monitoring;

- Sustainable use;

- Preservation and improvement of environment quality and reconstruction of deteriorated areas;

- Creation of a participation frame for the NGOs and population in the decisions' elaboration and application;

- Development of international co-operation for environment quality ensuring. The protection of touristic patrimony is largely influenced also by the population's ecological conscience and their respect for nature, historic sights and art and architecture monuments created along time. Tourists' education is absolutely necessary for nature's protection. In our country there are 586 protected sights and areas (4.8\% of the country's territory), very many situated in the mountainous area. Among them, a particular interest is raised by national parks, which, according to statistics, are 12 in number, completed by two biosphere reservations. Although they were declared as such, these protected areas do not operate at presents as biosphere reservations or national parks, the touristic activities do not take place within them according to precise ecological principles. That is why we consider that it is absolutely necessary to approach the tourism activity in these areas from the perspective of sustainable development, more precisely, an approach of the national park touristic product from the marketing perspective.

As shown before, most national parks in Romania are situated in the mountains, and thus we can consider the tourism generated by the national park touristic product as a form of mountainous tourism. Defining the strategies appropriate for this form of tourism in Romania, both on the macroeconomic and microeconomic level, should start from a good awareness of the mountainous touristic potential (in general) and of the areas considered (national parks - in particular), of the current degree of valuing, as well as of the economic, social and political conjecture influencing the evolution of 
Romanian tourism in general and of mountainous tourism in particular. (Costencu, 2008)

A useful analysis model, as basis for establishing development strategies, is constituted by the S.W.O.T. matrix. This model reunites a set of information related to the present state of the mountain tourism market, allowing the identification of adequate strategies. The model supposes:

- a market analysis highlighting the strengths and weaknesses of this form of tourism;

- an analysis of the environment external to the market, from which the opportunities and limitations or threats against its development will result.

- If we attempt to synthesise these analysis components, we can present as follows:

\section{Strengths:}

- An extremely valuable touristic potential, of great complexity and variety, with numerous endemic species, on a relatively wide territory; a mostly virgin nature;

- A fortunate combination, in many areas, of proper mountain touristic resources with natural therapeutic factors (mineral, thermal or saline springs, sedative microclimate), which allows the arrangement or development of complex-profile resorts;

- High accessibility, as in most areas there are large and populated valleys, together with an intense communication network - roads and railways - allowing access from all directions towards the destination areas;

- The existence of a very valuable cultural-religious patrimony, heritage of the Romanian spirituality, which contributes to the originality of the Romanian mountainous area in the European space;

- Still numerous people live in the valleys adjacent to the peripheral zones of national parks, population that is welcoming and interested in the development of touristic activities;

- A natural environment generally deprived of major pollution sources;

- Very important and increasing touristic potential demand (especially the domestic one) motivated by the possibility of practising different forms of tourism in the mountain area;

- Generally low prices and tariffs compared to the foreign tourists' purchasing power ;

- Marketing communication network already operational in Romania and abroad, including Romanian agencies and offices for tourism promotion.

Although these advantages are real and obvious, there are, unfortunately, very many weaknesses manifested in this field, among which:

- Unequal spatial distribution of natural touristic resources, from the viewpoint of structure, volume, value and possibility of valuing;

- The lack of a precise evaluation of potential touristic resources;

- The network of accommodation and food supply touristic units of medium and reduced comfort in the touristic nuclei in the communities living at the periphery of national parks, as well as in the units of recreation and treatment, which leads, on the whole, to a inadequate quality of services in these areas;

- Weak technical performances of set-ups for this form of tourism;

- Unequal repartition of touristic investments, of set-up efforts, most areas with valuable potential remaining unvalued;

- Poor maintenance of most access roads to the mountain area (where most national parks lie), especially of the secondary roads and mountain tracks;

- Reduced range of touristic sub-products promoted both on the domestic and the external market; 
- Visible degradation of the natural environment in the domestic circulation areas, due to soil erosion a well as neglect of visitors in the respective areas, lack of education in the spirit of natural protection.

There are thus a series of drawbacks of tourism activity in the mountain area, in general, in national parks in particular, and thus there are many things to do in view of a sustainable exploitation of protected areas.

Some opportunities for the development of this form of tourism may be:

- A certain saturation on the market of traditional destinations, which may constitute an advantage if we develop and promote an original and competitive offer;

- A relative growth of the interest in this respect, which can determine a more important attraction of investments in the arranging and equipping national parks for touristic purposes.

Among the threats and limitations hindering the development of tourism in protected areas we can mention:

- $\quad$ Strong competition on the European market (high number of national parks and natural reservations in Europe);

- Rather low interest manifested by the authorities in the touristic valuing of protected areas;

- Poor involvement of local communities in the touristic development, due to the lack of experience in this domain;

- Absence of an adequate general and touristic infrastructure.

All these elements should be taken into consideration when establishing the global conception for the higher valuing of these areas.

\section{Conclusions}

Aligning this particular touristic product to the trends of supply and demand manifested for this form of tourism - or for the mountain tourism in general - on the international plane is a real necessity. Consequently, the objectives, strategies and concrete actions taken for the macroeconomic and territorial development of tourism in national parks, as well as in the touristic nuclei situated in the communities living at the periphery of protected areas should permanently aim at supporting these trends. The destination areas should meet the economic, social and ecological requirements in competition, so that they observe the integrity of natural resources and of local communities. The upper limit of the number of allowed visitations is intentionally set at a level below the identified level of accepted use, so that in the long run the environment should be able to cover the possible increases appeared in the number of tourists, without any harm inflicted to the environment.

\section{References}

Butler, R.W. (1990), The concept of tourist area cycle of evolution: implications for management of resources, The Canadian Geographer, 24.

Costencu, M. (2007), Ecotourism. The national park touristic product, Timişoara, Edition Mirton.

Costencu, M. (2008), Ecotourism marketing. Strategies of the national park touristic product, Timişoara, Mirton Edition.

Fennel, D.A. (1999), Ecotourism - an introduction, London, Routledge.

Oarcea, Z, (1990), Studies of systematisation and organisation of the Cerna Valley Domogled National Park. 
Smaranda, J.S., (2008), Tourism management in protected natural areas, Cluj Napoca, Risoprint Edition.

Ţigu, G, (2005) coord., Resources and touristic destinations throughout the world, Bucharest, Editions Uranus.

Wearing, S., Neil, J, (2000), Ecotourism - Impacts, Potentials and Possibilities, Great Britain, Reed Educational and Professional Publishing Ltd. 